ORIGINAL ARTICLE

\title{
Cost effectiveness of adding magnetic resonance imaging to the usual management of suspected scaphoid fractures
}

\author{
S Brooks, F M Cicuttini, S Lim, D Taylor, S L Stuckey, A E Wluka
}

Br J Sports Med 2005;39:75-79. doi: 10.1136/bjsm.2003.007435

See end of article for authors' affiliations

.....................

Correspondence to: Dr Wluka, Department of Epidemiology and Preventive Medicine, Monash University, Alfred Hospital, Commercial Road, Prahran, Victoria 3181, Australia; anita.wluka@ med.monash.edu.au

Accepted 4 February 2004

\begin{abstract}
Objective: To determine the cost effectiveness of a magnetic resonance imaging scan (MRI) within 5 days of injury compared with the usual management of occult scaphoid fracture.

Methods: All patients with suspected scaphoid fractures in five hospitals were invited to participate in a randomised controlled trial of usual treatment with or without an MRI scan. Healthcare costs were compared, and a cost effectiveness analysis of the use of MRI in this scenario was performed.

Results: Twenty eight of the 37 patients identified were randomised: 17 in the control group, 11 in the MRI group. The groups were similar at baseline and follow up in terms of number of scaphoid fractures, other injuries, pain, and function. Of the patients without fracture, the MRI group had significantly fewer days immobilised: a median of 3.0 (interquartile range 3.0-3.0) $v 10.0(7-12)$ in the control group $(p=$ 0.006). The MRI group used fewer healthcare units (median 3.0, interquartile range 2.0-4.25) than the control group (5.0, 3.0-6.5) ( $p=0.03$ for the difference). However, the median cost of health care in the MRI group (\$594.35 AUD, \$551.35-667.23) was slightly higher than in the control group (\$428.15, $\$ 124.40-702.65$ ) ( $p=0.19$ for the difference). The mean incremental cost effectiveness ratio derived from this simulation was that MRI costs $\$ 44.37$ per day saved from unnecessary immobilisation $195 \%$ confidence interval $\$ 4.29$ to $\$ 101.02$ ). An illustrative willingness to pay was calculated using a combination of the trials measure of the subjects' individual productivity losses and the average daily earnings.

Conclusions: Use of MRI in the management of occult scaphoid fracture reduces the number of days of unnecessary immobilisation and use of healthcare units. Healthcare costs increased non-significantly in relation to the use of MRI in this setting. However, when productivity losses are considered, MRI may be considered cost effective, depending on the individual case.
\end{abstract}

$\mathrm{T}$ he scaphoid bone is the most commonly fractured carpa bone, accounting for $51-90 \%$ of carpal fractures and $2-7 \%$ of all fractures. ${ }^{1}$ However, scaphoid fractures are difficult to diagnose accurately because often the initial radiographs show no sign of fracture. ${ }^{2}$ A missed scaphoid fracture is likely to have an adverse outcome because the scaphoid has a relatively poor blood supply, particularly to its proximal pole. If untreated, healing may be delayed or result in non-union, malunion, or avascular necrosis. ${ }^{3}$ Consequently, patients presenting with a clinically suspected scaphoid fracture, but negative initial radiographs, are treated with temporary cast immobilisation until, at about two weeks, another imaging modality is used in an attempt to obtain an accurate diagnosis. ${ }^{1}$

Magnetic resonance imaging (MRI) is at least as accurate as other modalities for diagnosing scaphoid fractures as it produces highly detailed images and has the additional benefit of being able to diagnose fractures earlier after trauma than other modalities, such as radiographs, skeletal scintigraphy, and computed tomography scans. ${ }^{4}$ MRI can be used to diagnose suspected scaphoid fractures as early as 2.8 days after injury as opposed to about two weeks for other modalities. ${ }^{5}$ Consequently, patients who do not have a fracture (about $50-75 \%$ of patients with initially suspected scaphoid fractures) may avoid significant, unnecessary immobilisation time with the use of MRI. ${ }^{5}$ Although the present cost of MRI is often thought to be too great to warrant its use in the diagnosis of suspected scaphoid fractures, it has been suggested that it may be cost effective if unnecessary plaster cast immobilisation is avoided. ${ }^{6}$

We performed a study to determine if the addition of early, limited wrist MRI to the usual management of suspected occult scaphoid fractures would improve patient management, by improving patient outcomes and minimising costs to the healthcare system and community, in the Australian healthcare system.

\section{METHODS}

We performed a non-blinded, randomised controlled trial of the effect of the addition of MRI performed early in the management of suspected (occult) scaphoid fractures. Subjects were recruited through the emergency departments of five major city and suburban hospitals using the emergency department or radiology triage, or electronic log. All eligible patients presenting to the emergency departments between 1 January 2000 and 31 December 2002 were included. The study was approved by the ethics committees of all participating hospitals. All subjects gave written informed consent.

Inclusion criteria were: age greater than 18 years, presentation with a clinical diagnosis of possible occult scaphoid fracture requiring immobilisation with normal and/ or inconclusive initial wrist radiographs. Subjects were excluded if they had any contraindications to MRI (pacemaker, cerebral aneurysm clip, cochlear implant, presence of metal/shrapnel in strategic locations such as the eye, or claustrophobia) or were unable to cooperate with study requirements and provide informed consent. Once consent was obtained, subjects were randomised using computer generated random numbers to receive either MRI or no MRI

Abbreviations: $I Q R$, interquartile range; $M R I$, magnetic resonance imaging 
Table 1 Baseline characteristics of randomised groups

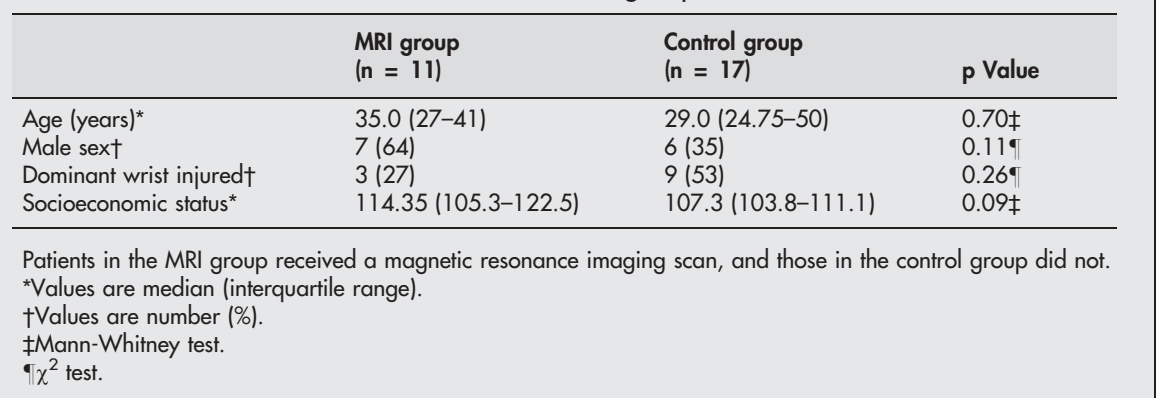

in a ratio of $1: 1$. Block randomisation, based on each hospital as a unit of randomisation was used.

Patients in the intervention arm had an MRI scan of the wrist within two to five days of presenting to the emergency department. The results of the scan were given to the patient, the doctor managing the patient, or a doctor specified by the patient. No advice was given to the patient to change their treatment in any way. Apart from MRI in the intervention group, no other alterations to the treatment were made and the treating doctor remained in charge of all management. Subjects in the control group had "usual care" as determined by their treating medial practitioners. The treating medical practitioners determined all further management of the patient with no involvement of the study team.

Wrist MRI was performed on a $1.5 \mathrm{~T}$ whole body magnetic resonance unit (Signa Horizon HiSpeed GE Medical Systems, Milwaukee, Wisconsin, USA, or equivalent), with the use of a commercial extremity coil. Total data acquisition time was 7-10 minutes per subject, with the longest individual sequence taking five minutes. The following imaging sequences were used: coronal TIW-SE (spin echo) and coronal STIR (inversion recovery). To avoid inaccurate results caused by inexperience, the scans were assessed by a senior radiologist at each site, with copies sent to a central reading site, where two readers reviewed the study, reaching a consensus in cases of discrepancy. Our criteria for fracture were the presence of any signal abnormality, be it oedema or a linear one suggesting macrofracture. Subjects in the control arm did not have their management altered in any way. The diagnosis was based on the modality or modalities that their treating doctor used.

All the subjects were contacted by telephone at baseline and then by telephone or post monthly for three months to collect information on resource use and recovery of wrist function and pain using the validated patient rated wrist evaluation. ${ }^{7}$ Resource use included the type and number of units of healthcare used as follows: visits to general practitioner, emergency department, specialist medical provider, physiotherapist, or "other" healthcare provider, which included other specialists such as a naturopath or remedial massage practitioner. The baseline questionnaire also gathered information on possible covariates (age, sex, occupation, socioeconomic status, and the type of injury), duration of time in splint, time off work, time during which unable to perform daily activities.

Data were collected on self coding forms and entered into a Microsoft Access database. The basic characteristics and distribution of prognostic variables were compared between the two groups. All proportions were analysed using $\chi^{2}$ statistics. Owing to the small sample size and non-normality of the data, variables were reported as the median and interquartile range (IQR). Variables were analysed using the Mann-Whitney test for non-parametric data. An exception to this was the analysis of the patient rated wrist evaluation.

A cost effectiveness analysis, using the method outlined below, was performed to determine the most appropriate management regimen for diagnosing suspected scaphoid fractures. Current practice was compared with current practice plus wrist MRI. All health system costs that were expected to vary between the two groups were collected. Total healthcare use included emergency department visits, general practitioner consultation, specialist (initial and subsequent consultations) physiotherapy, and the cost of diagnostic services (radiographs, skeletal scintigraphy, and MRI). We did not include the initial emergency department visit and initial radiograph, as these were performed on all subjects before randomisation (table 3 ). The differences in expenditure between the two groups were determined from the costs of healthcare use. The costs of health services used were derived from the Medicare Benefits Schedule, 2002. ${ }^{8}$ All costs were measured in Australian dollars (AUD).

The effectiveness measure was the number of days subjects unnecessarily spent in plaster, as this was the most sensitive measure available. To determine the cost effectiveness of

Table 2 Comparison between MRI and control group of clinical end points

\begin{tabular}{|c|c|c|c|}
\hline & $\begin{array}{l}\text { MRI group } \\
(n=11)\end{array}$ & $\begin{array}{l}\text { Control group } \\
(n=17)\end{array}$ & p Value* \\
\hline Number of scaphoid fractures $\ddagger$ & $3(27.3)$ & $4(23.5)$ & $0.82 \dagger$ \\
\hline Number of other fractures $\ddagger$ & $4(36.3)$ & $2(11.8)$ & $0.12 \dagger$ \\
\hline Days until diagnosis & $3.0(3.0-3.0)$ & $10.0(10.0-12.0)$ & 0.003 \\
\hline Days unnecessarily in plaster & $0.00(0.0-3.0)$ & $7(0-12.0)$ & 0.029 \\
\hline Days unnecessarily in plaster (subjects without fracture only) & $3.0(3.0-3.0)$ & $10.0(7-12)$ & 0.006 \\
\hline Days off work & $1.0(0.0-2.0)$ & $1.0(0.0-4.0)$ & 0.31 \\
\hline Activities unable to perform ( $1-5$ scale) & $2.0(2.0-5.0)$ & $3.5(2.0-5.0)$ & 0.69 \\
\hline Equivalent percentage & $10-25 \%(10-25 \%$ to $>75 \%)$ & $37-50 \%(10-25 \%$ to $>75 \%)$ & 0.69 \\
\hline
\end{tabular}

Patients in the MRI group received a magnetic resonance imaging scan, and those in the control group did not.

*Mann-Whitney test, unless otherwise noted.

$+\chi^{2}$ test.

$\ddagger$ Values are number (\%).

-Values are median (interquartile range) 
Table 3 Total healthcare use

\begin{tabular}{|c|c|c|c|c|c|}
\hline \multirow[b]{2}{*}{ Healthcare resources } & \multirow{2}{*}{$\begin{array}{l}\text { Cost } \\
\text { (AUD) }\end{array}$} & \multicolumn{2}{|l|}{ MRI group } & \multicolumn{2}{|l|}{ Control group } \\
\hline & & Units & Total cost of service (AUD) & Units & Total cost of service (AUD) \\
\hline \multicolumn{6}{|l|}{ General services } \\
\hline Emergency department visit & 24.45 & 1 & 24.45 & 1 & 24.45 \\
\hline General practitioner consultation & 24.45 & 8 & 195.6 & 24 & 568.8 \\
\hline Specialist (initial consultation) & 119.35 & 7 & 835.45 & 9 & 1074.15 \\
\hline Specialist (subsequent consultations) & 59.75 & 4 & 239 & 16 & 956 \\
\hline Physiotherapist & 40 & 0 & 0 & 8 & 320 \\
\hline Total general services per person* & & $2.0(1.0-3.0)$ & $119.35(67.24-185.21)$ & $3.0(2.0-4.5)$ & $228.0(48.90-63.30)$ \\
\hline \multicolumn{6}{|l|}{ Diagnostic imaging services } \\
\hline Radiographs & 28.05 & 3 & 84.15 & 14 & 392.7 \\
\hline Skeletal scintigram & 295.1 & 0 & 0 & 4 & 1180.4 \\
\hline MRI & 475 & 10 & 4750 & 4 & 1900 \\
\hline Total diagnostic services per person $†$ & & $1.0(1.0-2.0)$ & $475.00(475.00-503.05)$ & $1.0(1.0-2.0)$ & $295.10(28.05-475.00)$ \\
\hline Total units/expenditure per person $\ddagger$ & & $3.0(2.0-4.25)$ & $594.35(551.05-667.23)$ & $5.0(3.0-6.5)$ & $428.15(124.40-702.65)$ \\
\hline \multicolumn{6}{|c|}{ 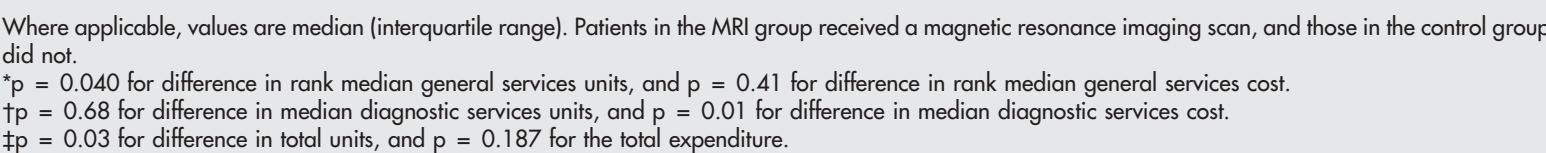 } \\
\hline
\end{tabular}

adding MRI and to assess the uncertainty around the cost effectiveness ratio, the bootstrap method (1000 simulations), as described by Briggs and Fenn, ${ }^{9}$ was performed. In contrast with previous analyses, this considered mean values as recommended by Briggs and Fenn. ${ }^{9}$ The simulation data were used to determine a mean incremental cost effectiveness ratio. ${ }^{9}$

A scatter plot was produced to illustrate the results of the simulation, and an acceptability curve was produced to show the probability that MRI is cost effective according to a range of willingness to pay thresholds. ${ }^{9}$ To estimate an appropriate willingness to pay per day saved in plaster, the following calculation was used:

$$
\mathrm{WP}=\mathrm{PL} \times \mathrm{DE}
$$

where WP = willingness to pay, $\mathrm{PL}=$ productivity loss due to immobilisation (\%), and DE = daily earnings.

ICER scatter plot: what does the postion of the data mean?

\begin{tabular}{|c|c|}
\hline $\begin{array}{l}\text { MRI is more expensive } \\
\text { MRI results in more days in } \\
\text { unnecessary plaster }\end{array}$ & $\begin{array}{l}\text { MRI is more expensive } \\
\text { MRI results in fewer } \\
\text { unnecessary days in plaster }\end{array}$ \\
\hline $\begin{array}{l}\text { MRI is less expensive } \\
\text { MRI results in more days in } \\
\text { unnecessary plaster }\end{array}$ & $\begin{array}{l}\text { MRI is less expensive } \\
\text { MRI results in fewer } \\
\text { unnecessary days in plaster }\end{array}$ \\
\hline
\end{tabular}

Days saved in plaster

B

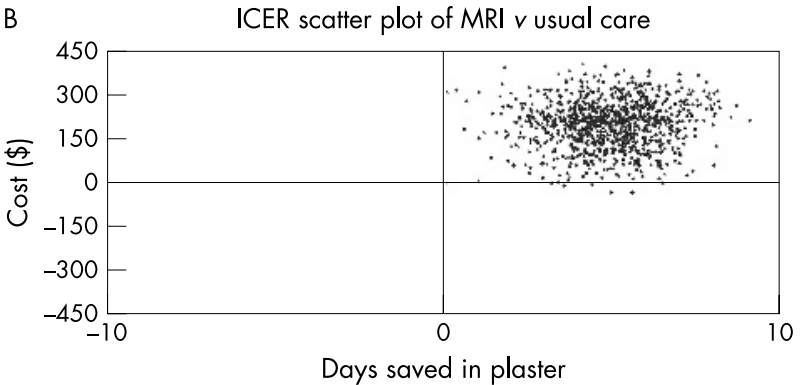

Figure 1 Scatter plot illustrating the relevance of the position of data points (A) and incremental cost effectiveness ratio (ICER) scatter plot of simulation data (B). Of note is the fact that most of the data points are within quadrant 1 , indicating that the use of magnetic resonance imaging (MRI) costs more but with increased effectiveness as defined by number of days saved from unnecessary plaster immobilisation.
To illustrate the possible cost effectiveness of adding MRI, this calculation was combined with the trial results to produce a range of possible willingness to pay values. An appropriate range of productivity losses (PL) was determined from the IQR of the percentage of daily activities that trial subjects were unable to perform (reported on questionnaire). A single average daily earning was used in the calculation of the willingness to pay range. This was obtained from the average Australian wage (full time adult ordinary time earnings \$868.50/week) obtained from the Australian Bureau of Statistics (May 2002).

Sample size was estimated on the basis of the estimated difference in days unnecessarily immobilised, so that, with a mean number of days unnecessarily in plaster in the MRI group of 3, and 10 (SD 3) days being the mean in the control group, with five subjects in each group, the study had a power of $80 \%$ to detect a significant difference, with an $\alpha$ error of 0.05 .

\section{RESULTS}

Thirty seven potential subjects from the emergency triage or electronic log, treated for a suspected scaphoid fracture, were identified. Twenty eight agreed to participate in the study, six subjects declined to participate, two subjects could not be contacted, and one agreed to participate but moved overseas before any study involvement. Of the participating subjects, 11 were randomised to the "MRI" group, and 17 to the

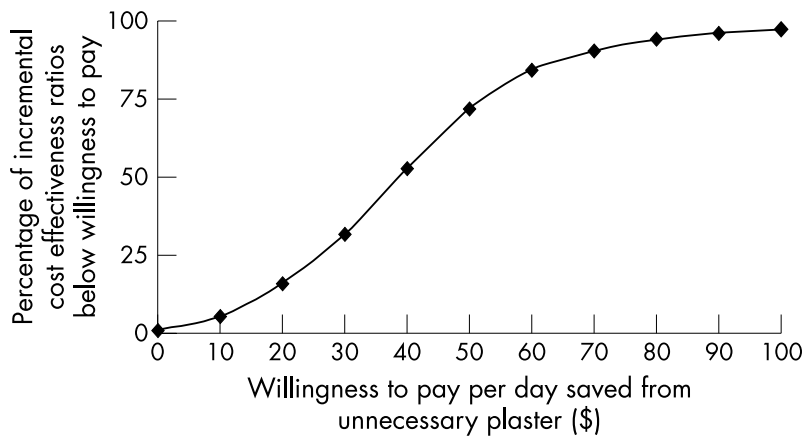

Figure 2 Acceptability curve illustrating the proportion of simulations below a range of willingness to pay values. At a cost of $\$ 50$ per day saved from unnecessary cast immobilisation, the introduction of magnetic resonance imaging will be cost effective $70 \%$ of the time. 
Table 4 Illustrative range of willingness to pay and corresponding possible cost effectiveness

\begin{tabular}{lll}
\hline $\begin{array}{l}\text { Productivity } \\
\text { loss (\%) }\end{array}$ & $\begin{array}{l}\text { Willingness to pay } \\
\text { (AUD) }\end{array}$ & $\begin{array}{l}\text { Percentage of cost effective } \\
\text { ICERs }\end{array}$ \\
\hline 10 & 17.37 & 11.7 \\
50 & 86.85 & 95.3 \\
75 & 130.28 & 98.7 \\
\hline
\end{tabular}

ICER, Incremental cost effectiveness ratio.

"control" group. One subject from the MRI group was lost to follow up after moving house and becoming uncontactable.

The groups were similar at baseline (table 1). Although there were more men, more dominant wrist injuries, and higher socioeconomic status in subjects in the MRI group, these differences were not significant (table 1).

The percentages of definite scaphoid and non-scaphoid fractures diagnosed were similar in the two groups (table 2). Scaphoid fracture was eliminated or confirmed significantly more quickly in the MRI group (median 3 days, IQR 3-3) than in the usual treatment group (median 10 days, IQR 10$12 ; p=0.003)$ (table 2). The main clinical difference between the groups was that, when only subjects diagnosed as having no fracture were included in the analysis, the median number of days unnecessarily in plaster in the MRI group was three days, which is significantly less than the median of 10 days in the control group $(p=0.006)$ (table 2 ).

The change in quality of life caused by being in plaster for a scaphoid fracture was quantified by measuring the days of work lost and estimated disruption to daily activities. There was no difference in the number of days of work missed (table 2). At baseline, the two groups showed similar high levels of pain and impaired function, as measured by the patient rated wrist evaluation: in the MRI group the mean (SD) pain and function scores were $5.9(0.07)$ and $7.08(0.70)$ compared with $6.5(0.56)$ and $8.03(0.56)$ in the control group. The two groups showed a similar, significant decrease in both pain and functional rating over the three month period $(\mathrm{p}<0.001)$. There were no significant differences in pain or function between the MRI and control groups one month, two months, and three months after injury.

The differences in all costs associated with diagnosis, treatment, and management of the suspected scaphoid fracture between the two groups were determined from the costs of healthcare use (table 3). There was a trend towards a higher cost for the MRI group than the control group (median of \$594.35 compared with \$428.15). However, the IQRs of the two groups were quite different. The MRI group had a narrow range of \$551.05-667.23 compared with a wide range for the control group of \$124.40-702.65. Healthcare use was lower in the MRI group, with a median of 3.0 units (IQR 2.0-4.25), compared with the control group, with a median of 5.0 (IQR 3.0-6.5) ( $\mathrm{p}=0.03$ for the difference).

\section{COST ANALYSIS}

The mean incremental cost effectiveness ratio derived from this simulation was $\$ 44.37$ per day saved from unnecessary immobilisation by the use of MRI (95\% CI $\$ 4.29$ to $\$ 101.02$ ). The scatter plot of incremental cost effectiveness ratios (fig l) illustrates that most simulations are in quadrant 1 , indicating an increased cost for MRI but with increased effectiveness. ${ }^{9}$ This relates to fewer days unnecessarily spent in plaster for the subjects who had an MRI scan. In fact, a small percentage of simulations appear in quadrant 2, which indicates an increased effectiveness of MRI with a decreased cost-that is, MRI is both cost saving and more effective than "usual care". ${ }^{\prime}$ An acceptability curve (fig 2 ) was plotted to illustrate the proportion of incremental cost effectiveness ratios that are cost effective over a range of costs that may be considered acceptable for the benefit of days saved from unnecessary immobilisation.

An illustrative willingness to pay was calculated from the trial results using a combination of the trials measure of the subjects' individual productivity losses (table 3 ) and the average daily earnings of $\$ 173.70$ based on the Australian Bureau of Statistics average earnings for 2002 (full time adult ordinary time earnings: $\$ 868.50 \mathrm{AUD} /$ week). The IQR of the percentage of activities that patients were unable to perform was used to develop the range of possible willingness to pay values (table 4). This range indicated that, at a 50\% productivity loss, the addition of MRI was a cost effective addition in most cases (95.3\%). At a very minor productivity loss $(10 \%)$, the cost effectiveness was greatly reduced so that MRI was cost effective in only $11.7 \%$ of simulations.

\section{DISCUSSION}

We have found in an unblinded, randomised controlled trial of normal adults with suspected scaphoid fractures, that the addition of an MRI scan within five days of injury to the usual care reduced the days unnecessarily spent immobilised in a plaster cast and the use of healthcare services. Despite the decreased use of services by those who had an MRI scan in the first few days, the total expenditure in the two groups was similar. However, a cost effectiveness analysis suggested that the early addition of MRI may be cost effective if the high productivity losses associated with unnecessary immobilisation are included.

Our findings of a reduction in the time until diagnosis in the MRI group are not unexpected. It has been shown that an MRI scan can clarify the diagnosis in subjects in whom radiographs are normal, less than 42 hours after injury. ${ }^{10}$ Despite this, most studies recommend that repeat radiographs be performed two weeks after injury, but even then the diagnosis of scaphoid fracture may not be confidently excluded and additional imaging may be required. ${ }^{411}{ }^{12}$ It has been suggested that skeletal scintigraphy be performed two weeks after injury. ${ }^{12}{ }^{13}$ However, these management strategies result in many patients being unnecessarily immobilised for at least 7-10 days until a diagnosis is made.

We showed that addition of MRI early in the care of suspected scaphoid fracture reduced use of healthcare units. This is similar to the results of Raby, ${ }^{10}$ who found that use of MRI early in the management of suspected scaphoid fracture reduced fracture clinic attendance compared with MRI use in a selected group, with continuing symptoms 10 days to 6 weeks after injury. However, this study did not account for any other healthcare use, whereas we have included the cost of these.

Despite the reduction in use of healthcare units seen with the addition of MRI early in the care of suspected scaphoid fracture, when this was examined in terms of cost, there was no significant difference between the two groups. This lack of difference could be explained by the high cost of MRI ( $\$ 475$ AUD). At present, in the Australian healthcare system, all MRI services cost the same irrespective of the body part or time required for the MRI. ${ }^{8}$ Should this funding change, with a reduction in cost for limited scans or dedicated extremity systems, there would be an improvement in the cost effectiveness of the use of MRI in this setting. ${ }^{14}$

It has also been suggested that MRI is likely to be more cost effective when productivity and income loss are considered. ${ }^{6}$ The latter study by Dorsay et al used modelling to compare current ideal recommendations in the management of an occult scaphoid fracture with management incorporating the addition of a screening MRI scan to investigate the theoretical costs of the various methods of diagnosing suspected scaphoid fractures. The results suggested 
what is already known on this topic

MRI is useful in the management of occult scaphoid fracture. It can be used to diagnose a fracture or other injury or exclude the presence of a fracture earlier than other imaging modalities. Models of MRI use in this situation have suggested that it may be cost effective, but this has not been fully examined.

that the impact on earnings would further add to the cost effectiveness of MRI in that study, but did not actually include earning losses or other associated costs of the scaphoid injury, such as associated healthcare use. ${ }^{6}$ Our study provides evidence to support these findings. The costs in the two groups were very similar. However, the MRI group also benefited from a mean of seven days less in plaster. Thus, addition of some value to the improved functioning over that time needs to be considered. The optimum way to do this is not clear. However, it should be considered when a decision is made about adding MRI to the management of an individual patient.

Our cost effectiveness simulation indicates that MRI costs $\$ 44.37$ (95\% CI $\$ 4.29$ to $\$ 101.02$ ) per day saved of unnecessary immobilisation. Whether this is acceptable or not depends on what society considers an appropriate amount to pay for the possibility of days saved from plaster. Our data suggest that, at the median productivity loss (50\%), the addition of MRI would be cost effective $95.3 \%$ of the time. However, this example only used one measure of earnings, the average Australian wage, to calculate the willingness to pay value. Individual patients will be affected to varying degrees by the unnecessary immobilisation and similarly they will have a range of earnings. Using our results, a patient can use this acceptability curve combined with the willingness to pay equation to determine whether MRI is a cost effective option in their case. For example, a self employed manual labourer, earning \$350 AUD per day, dependent on being physically unimpaired, would have a higher willingness to pay-for example, impaired 10\%, willingness to pay \$35 AUD per day-than a worker on the average Australian wage. Using the accessibility curve, in this situation, MRI is likely to be cost effective in about $40 \%$ of cases.

The main limitation of our study is the small number of subjects. Although scaphoid fractures are considered relatively common, the number of eligible subjects was lower than expected. This has been the experience of other investigators also. ${ }^{1}$ In addition, a large number of subjects identified were younger patients (12-18 years), who were excluded from the study to avoid confounding related to the difference in healing of fractures in fully grown and developing bones. Although some may have been fully grown, subjects in this age group are likely to be dependents, and not yet in the workforce. Excluding them allowed continuity when the economic evaluation was performed. Despite this, we were able to detect a large difference between the two groups, such as days in plaster. As we used all the consecutive eligible subjects at five different city and suburban hospitals, both private and public, our results can be generalised to healthy adults with an occult scaphoid fracture. A strength of our study is that we used a very rigorous definition of occult scaphoid fracture. The clinical suspicion had to be strong enough that immobilisation was used in the absence of an abnormal radiograph. Although our study was not blinded, outcome measures were assessed
What this study adds

This study confirms the usefulness of MRI in the management of occult scaphoid fracture, as it reduces the time spent unnecessarily immobilised by patients without fractures. It also shows that the addition of MRI reduces healthcare use and is cost effective at modest levels of individual willingness to pay to avoid unnecessary immobilisation.

objectively using a validated instrument and standard questions by trained observers to minimise bias. Although the study was performed in Australia, we have reported our findings in healthcare units. This enables interpretation of our study findings in other settings.

In summary, our study suggests that the addition of wrist MRI to the management of occult scaphoid fracture reduces the number of days the patient is immobilised in a plaster cast and the use of healthcare units. However, on the basis of current funding for MRI in Australia, there was a nonsignificant increase in the healthcare costs incurred. Our data suggest a mean incremental cost effectiveness ratio of $\$ 44.37$ (95\% CI \$4.29 to \$101.02), being an estimate of how much extra MRI costs for the days it saves from unnecessary plaster cast immobilisation. Studies such as this provide data from which decisions can be made about whether to use MRI in the management of occult scaphoid fracture.

\section{ACKNOWLEDGEMENTS}

This study was funded by the Consultative Committee on Diagnostic Imaging. AEW is the recipient of an NHMRC scholarship and additional funds from the Alfred Trusts.

\section{Authors' affiliations}

S Brooks, S Lim, D Taylor, F M Cicuttini, A E Wluka, Department of Epidemiology and Preventive Medicine, Monash University, Prahran, Victoria, Australia

S L Stuckey, MRI Department, Alfred Hospital, Prahran

Competing interests: none declared

\section{REFERENCES}

1 Tiel-van Buul MM, Roolker W, Broekhuizen AH, et al. The diagnostic management of suspected scaphoid fracture. Injury 1997;28:1-8.

2 Mittal RL, Dargan SK. Occult scaphoid fracture: a diagnostic enigma. J Orthop Trauma 1989:3:306-8.

3 Goldfarb CA, Yin Y, Gilula LA, et al. Wrist fractures: what the clinician wants to know. Radiology 2001;219:11-28.

4 Hunter JC, Escobedo EM, Wilson AJ, et al. MR imaging of clinically suspected scaphoid fractures. AJR Am J Roentgenol 1997; 168:1287-93.

5 Gaebler C, Kukla C, Breitenseher M, et al. Magnetic resonance imaging of occult scaphoid fractures. J Trauma 1996;41:73-6.

6 Dorsay TA, Major NM, Helms CA. Cost-effectiveness of immediate MR imaging versus traditional follow-up for revealing radiographically occult scaphoid fractures. AJR Am J Roentgenol 2001;177:1257-63.

7 MacDermid JC, Turgeon T, Richards RS, et al. Patient rating of wrist pain and disability: a reliable and valid measurement tool. J Orthop Trauma 1998; 12:577-86.

8 Medicare. Medicare benefits schedule 2002.

9 Briggs A, Fenn P. Confidence intervals or surfaces? Uncertainty on the costeffectiveness plane. Health Econ 1998;7:723-40.

10 Raby N. Magnetic resonance imaging of suspected scaphoid fractures using a low field dedicated extremity MR system. Clin Radiol 2001;56:316-20.

11 Brismar J. Skeletal scintigraphy of the wrist in suggested scaphoid fracture. Acta Radiol 1988;29:101-7.

12 Rayan GM. Fractures and nonunions of the scaphoid. J Okla State Med Assoc 1996;89:315-23.

13 Murphy D, Eisenhauer M. The utility of a bone scan in the diagnosis of clinical scaphoid fracture. J Emerg Med 1994;12:709-12.

14 Van Gelderen W, Gale RS, Steward AH. Short tau inversion recovery magnetic resonance imaging in occult scaphoid injuries: effect on management. Australas Radiol 1998;42:20-4. 